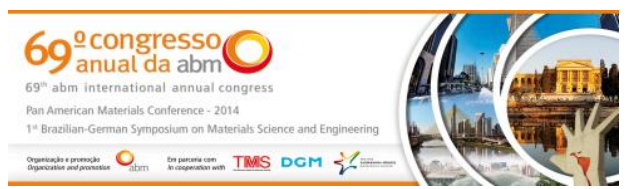

Tema: Materiais com efeito de memória de forma

\title{
COMPORTAMENTO MECÂNICO DE INSTRUMENTOS ENDODÔNTICOS DE Ni-Ti M-WIRE E Ni-Ti CONVENCIONAL ATRAVÉS DE SIMULAÇÃO PELO MÉTODO DOS ELEMENTOS FINITOS*
}

\section{Resumo}

Leandro de Arruda Santos ${ }^{1}$

Estevam Barbosa de Las Casas² Maria Guiomar de Azevedo Bahia ${ }^{3}$ Vicente Tadeu Lopes Buono 4

O objetivo deste trabalho foi avaliar o comportamento mecânico sob flexão de um instrumento endodôntico constituído de $\mathrm{Ni}$-Ti M-Wire e comparar suas respostas com aquelas obtidas de um instrumento de $\mathrm{Ni}$-Ti superelástico convencional com a mesma geometria, utilizando-se simulação por elementos finitos. Um instrumento ProTaper Universal F1 foi selecionado para este estudo. O modelo geométrico para a simulação numérica foi gerado através de micro-tomografia computadorizada e os dados para os modelos constitutivos foram obtidos por meio de ensaios de tração realizados em laboratório. A simulação numérica foi realizada em ABAQUS, com condições de contorno baseadas na especificação ISO 3630-1. Os resultados mostram que instrumentos de $\mathrm{Ni}-\mathrm{Ti} \mathrm{M}$-Wire apresentam maior flexibilidade $\mathrm{e}$ menores valores de tensão quando submetidos à flexão, o que indica que estes instrumentos são mais resistentes à fadiga por flexão.

Palavras-chave: Instrumentos endodônticos de Ni-Ti; M-Wire; Método dos elementos finitos.

\section{MECHANICAL BEHAVIOR OF M-WIRE Ni-Ti AND CONVENTIONAL Ni-Ti ENDODONTIC INSTRUMENTS VIA FINITE ELEMENT METHOD SIMULATION}

\section{Abstract}

The aim of this study was to evaluate the mechanical behavior under flexion of a $\mathrm{Ni}$ Ti M-Wire endodontic instrument and compare its responses with those obtained from a conventional superelastic $\mathrm{Ni}$ - $\mathrm{Ti}$ instrument with the same geometry using finite element simulation. A ProTaper F1 instrument was selected for this study. The geometric model for the numerical simulation was generated by micro-computed tomography and the data for the constitutive model were obtained by means of tensile tests performed in laboratory. The numeric simulation was performed in ABAQUS with boundary conditions that were based on the ISO 3630-1 specification. The results show that $\mathrm{M}-\mathrm{Wire} \mathrm{Ni}-\mathrm{Ti}$ instruments present higher flexibility and lower stress values when subjected to flexion what indicates that these instruments are more fatigue resistant.

Keywords: Ni-Ti endodontic instruments; M-Wire; Finite element method.

1 Engenheiro de Materiais, Doutor, Professor, Departamento de Engenharia Metalúrgica e de Materiais, Universidade Federal de Minas Gerais, Belo Horizonte, MG, Brasil.

2 Engenheiro Civil, Ph.D., Professor, Departamento de engenharia de Estruturas, Universidade Federal de Minas Gerais, Belo Horizonte, MG, Brasil.

3 Bacharel em Odontologia, Doutora, Professora, Departamento de Odontologia Restauradora, Universidade Federal de Minas Gerais, Belo Horizonte, MG, Brasil.

4 Físico, Doutor, Professor, Departamento de Engenharia Metalúrgica e de Materiais, Universidade Federal de Minas Gerais, Belo Horizonte, MG, Brasil.

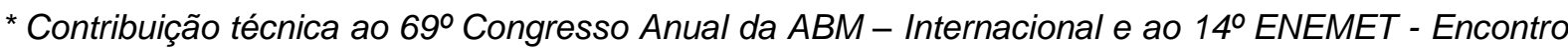
Nacional de Estudantes de Engenharia Metalúrgica, de Materiais e de Minas, 21 a 25 de julho de 2014, São Paulo, SP, Brasil.
} 


\section{INTRODUÇÃO}

A endodontia é o campo da odontologia que trata de infecções da polpa, tecido mole que está contido nos canais dentários. Limas endodônticas são utilizadas para formatar o canal quando sua obturação se torna necessária. Estes instrumentos devem apresentar uma boa flexibilidade e perfil de corte para penetrar no canal dentário e remover a dentina [1].

$\mathrm{O}$ advento de limas fabricadas com a liga $\mathrm{Ni}-\mathrm{Ti}$, de composição aproximadamente equiatômica, transformou os métodos de instrumentação de canais radiculares, pois esta liga permite a fabricação de instrumentos que mantêm a forma original do canal sem criar severas irregularidades [2]. Além disso, essas propriedades favoráveis permitiram a fabricação de limas de $\mathrm{Ni}$-Ti com novas geometrias em termos de secção transversal, conicidade e passo de rosca, além de permitir a introdução de movimentos rotatórios acionados a motor [3]. Essas características se devem principalmente à capacidade que as ligas $\mathrm{Ni}$-Ti possuem de recuperar deformações de até $\sim 10 \%$ após a retirada da carga. Esta capacidade é chamada de superelasticidade e se deve a uma transformação reversa no estado sólido entre a fase monoclínica de menor temperatura induzida por tensão, chamada martensita, e a fase de maior temperatura, austenita, cúbica do tipo B2. Essa transformação ocorre quando o material se encontra acima de uma temperatura específica, conhecida como $A_{\mathrm{f}}$ (austenite finish temperature), onde a austenita é mais estável. A superelasticidade presente nesta liga é uma característica que está associada ao efeito de memória de forma [4].

Embora a inserção de limas com Ni-Ti superelástico na prática clínica represente um importante desenvolvimento para a endodontia, estes instrumentos ainda podem sofrer falhas em serviço, principalmente em decorrência de fadiga por flexão. Durante a formatação de canais curvos, o instrumento rotatório é submetido a ciclos de tração e compressão na região de curvatura máxima do canal [5-7], podendo ocasionar a fratura do material por fadiga neste ponto [8, 9]. Quanto maior a curvatura do canal, mais severa é a condição de carregamento [5]. Logo, flexão é uma das condições de carregamento mais importantes às quais os instrumentos endodônticos são submetidos durante a prática clínica.

Com o objetivo de melhorar a resistência à fratura das limas de $\mathrm{Ni}-\mathrm{Ti}$, fabricantes introduzem novas técnicas de tratamentos termomecânicos de ligas $\mathrm{Ni}-\mathrm{Ti}$, otimizando assim características de sua microestrutura e verificando as consequências em termos de comportamento mecânico. Recentemente, um novo fio de Ni-Ti, comercializado sob a alcunha de M-Wire (Sportswire LLC, Langley, OK, EUA), foi desenvolvido para a fabricação de instrumentos endodônticos. Os parâmetros e condições deste tratamento não foram reveladas pelo fabricante, permanecendo desconhecidas. Trabalhos experimentais nessa liga já sugerem uma melhora significativa em termos de resistência à fadiga em comparação ao $\mathrm{Ni}-\mathrm{Ti}$ convencional [10].

O método dos elementos finitos é uma alternativa vantajosa no estudo do comportamento mecânico de instrumentos endodônticos, uma vez que permite a obtenção de informações que exigem caras e laboriosas práticas experimentais ou que não podem ser acessadas em laboratório (como a distribuição de tensões internas). Ademais, é possível utilizar uma mesma geometria de lima com diferentes materiais (modelos constitutivos), limitando o problema ao aspecto das diferentes propriedades do material e eliminando possíveis diferenças de desempenho devido a variações geométricas. Logo, o objetivo deste trabalho é contribuir no

\footnotetext{
* Contribuição técnica ao $69^{\circ}$ Congresso Anual da ABM - Internacional e ao 14ํㅡㄹ ENEMET - Encontro Nacional de Estudantes de Engenharia Metalúrgica, de Materiais e de Minas, 21 a 25 de julho de 2014, São Paulo, SP, Brasil.
} 


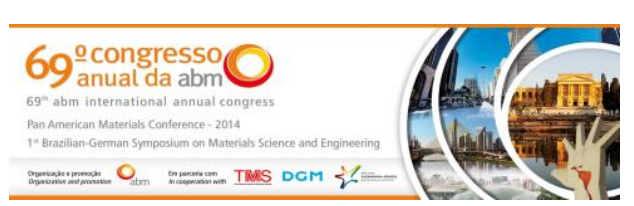

desenvolvimento de novas soluções para a terapia endodôntica através do uso da análise por elementos finitos para a comparação das propriedades mecânicas de limas fabricadas com $\mathrm{Ni}$-Ti convencional e $\mathrm{Ni}$-Ti $\mathrm{M}$-Wire e que possuem a mesma geometria.

\section{MATERIAIS E MÉTODOS}

A geometria do instrumento ProTaper Universal F1 (Dentsply Maillefer, Ballaigues, Suíça) foi selecionada para a elaboração do modelo geométrico tridimensional (3D). $O$ instrumento foi submetido à micro-tomografia computadorizada (eXplore Locus SP; GE Healthcare, Waukesha, WI, EUA) em intervalos de $12 \mu \mathrm{m}$. As imagens resultantes foram segmentadas (Mimics, Materialise $\mathrm{HQ}$, Leuven, Bélgica) para formar um modelo geométrico 3D. A haste do instrumento foi posteriormente inserida no modelo com o auxílio do software SolidWorks 2010 (three-dimensional computeraided design software, Concord, MA, EUA).

O modelo foi então malhado no software ABAQUS 6.9-1 (SIMULIA, Providence, RI, EUA) com elementos tetraédricos quadráticos de dez nós. A malha utilizada é não uniforme.

Fios de Ni-Ti convencional e de Ni-Ti M-Wire fornecidos pela Dentsply Maillefer foram submetidos a ensaios de tração até a ruptura em uma máquina de ensaios de tração Instron 5582 (Instron, Norwood, MA, EUA) em temperatura ambiente com uma taxa de $1 \times 10^{-3} \mathrm{~s}^{-1}$. Os parâmetros necessários para a elaboração do modelo constitutivo, utilizados como dados de entrada para a construção do modelo, foram extraídos das curvas resultantes dos ensaios de tração. O comportamento superelástico da liga pôde ser descrito com o auxílio de uma sub-rotina implementada e comercializada em ABAQUS, tendo como base o modelo analítico desenvolvido por Auricchio e Petrini [11].

As condições de contorno utilizadas neste estudo estão descrita na Figura 1 e tem como base ensaios mecânicos de flexão especificados pela norma ISO 3630-1 [12] para testes em limas endodônticas, não havendo demais interações ou contatos. Neste experimento uma lima é engastada (restrição de movimento em todas as direções) a $3 \mathrm{~mm}$ da ponta e flexionada através da haste até uma curvatura de $45^{\circ}$ em relação ao seu eixo longitudinal. Com um auxílio de uma célula de carga, o momento necessário para este movimento pode ser medido. Uma foto do experimento é exibida na Figura 2.

Todo o processo de simulação foi realizado com 0 auxílio do software ABAQUS 6.9-1.

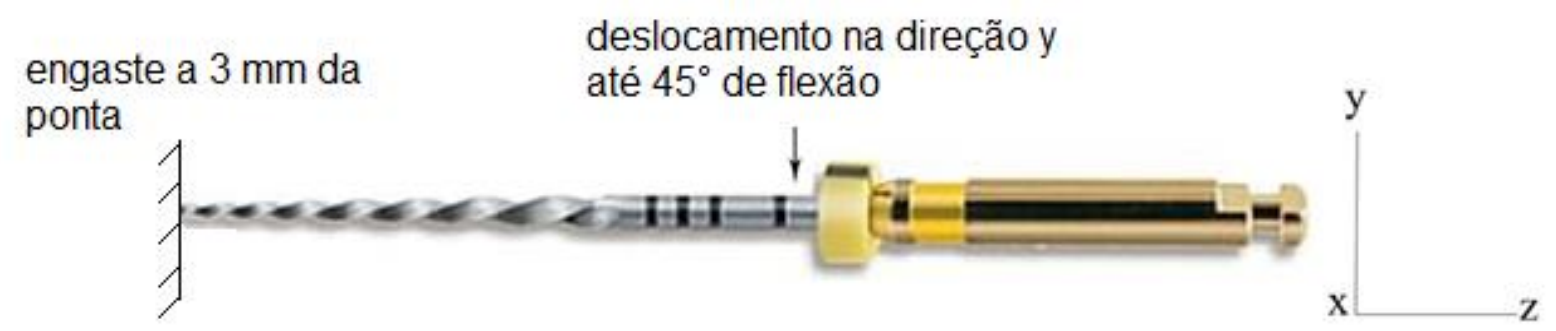

Figura 1. Condições de contorno para a simulação: flexão até $45^{\circ}$ com a lima ProTaper Universal F1 presa a $3 \mathrm{~mm}$ da ponta.

\footnotetext{
* Contribuição técnica ao $69^{\circ}$ Congresso Anual da ABM - Internacional e ao 14ํㅡㄹ ENEMET - Encontro Nacional de Estudantes de Engenharia Metalúrgica, de Materiais e de Minas, 21 a 25 de julho de 2014, São Paulo, SP, Brasil.
} 

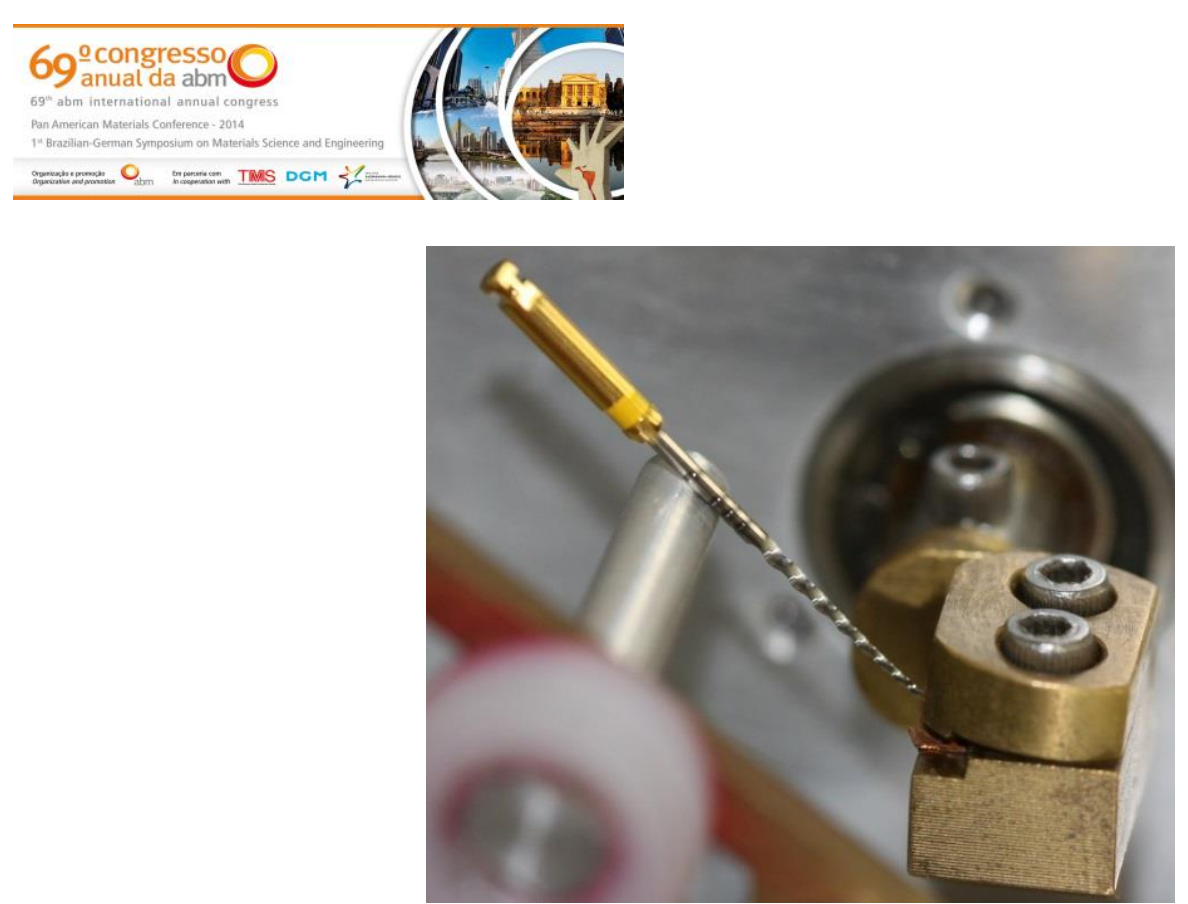

Figura 2. Ensaio experimental de flexão em uma lima ProTaper Universal F1.

\section{RESULTADOS E DISCUSSÃO}

A grande maioria dos trabalhos realizados sobre o comportamento mecânico de instrumentos endodônticos de $\mathrm{Ni}$ - Ti têm como base ensaios experimentais em laboratório [6, 7, 9, 10]. Entretanto, estes testes demandam extensivas horas laboratoriais e costumam ser onerosos, exigindo um grande número de amostras e o desenvolvimento de dispositivos adequados que atendam as normas internacionais de ensaios. A grande vantagem do estudo do comportamento desses instrumentos por simulação numérica está em obter-se informações com um número mínimo de amostras e em menor tempo. Experimentos em dispositivos de bancada podem ser reproduzidos através de condições de contorno e carregamento adequadas, além de informações sobre o estado de tensões internas do material poderem ser calculadas, algo que é impossível analiticamente. Evidentemente, a simulação numérica não substitui os ensaios experimentais, uma vez que os modelos de elementos finitos devem ser validados através da comparação direta com resultados experimentais para a verificação de sua acurácia, como realizado por Santos et al. [13]. Além disso, uma das etapas de confecção de um modelo numérico é a elaboração de um modelo constitutivo que faz uso dos resultados de ensaios mecânicos como dados de entrada. Ademais, todo modelo lança mão de simplificações que devem ser levadas em conta durante a análise dos resultados. Logo, a simulação numérica deve ser interpretada como uma alternativa a mais no estudo do comportamento mecânico de materiais, podendo ser utilizada em conjunto ou complementando os experimentos.

$\mathrm{Na}$ Figura 3 é exibido um esquema do processo de elaboração do modelo de elementos finitos para o instrumento ProTaper Universal F1. O processo de microtomografia computadorizada resultou em 1426 imagens da parte ativa do instrumento que foram segmentadas para a construção de um modelo geométrico tridimensional também mostrado na Figura 3. A importância da micro-tomografia neste trabalho reside no fato de que a geometria da parte ativa do instrumento possui uma complexidade bastante considerável, além de apresentar dimensões muito reduzidas. Assim, todos os detalhes geométricos do instrumento podem ser captados no modelo, aumentando a acurácia do mesmo. O cabo do instrumento pôde ser negligenciado no modelo, uma vez que não é submetido a qualquer

\footnotetext{
* Contribuição técnica ao $69^{\circ}$ Congresso Anual da ABM - Internacional e ao 14ํㅡㄹ ENEMET - Encontro Nacional de Estudantes de Engenharia Metalúrgica, de Materiais e de Minas, 21 a 25 de julho de 2014, São Paulo, SP, Brasil.
} 


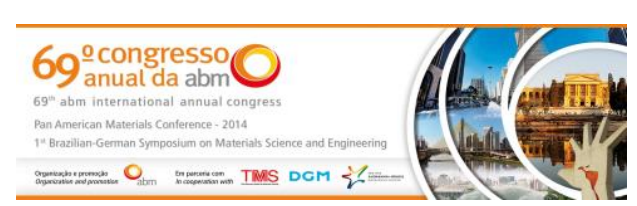

esforço durante o ensaio de flexão. O modelo de elementos finitos consistiu, por fim, de 51.184 elementos tetraédricos, com um total de 88.344 nós.

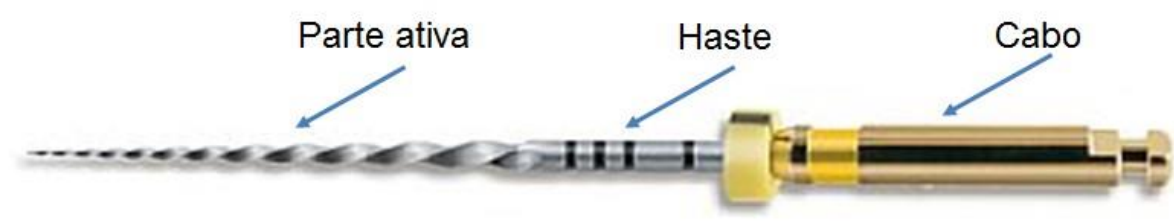

\section{Micro-tomografia}

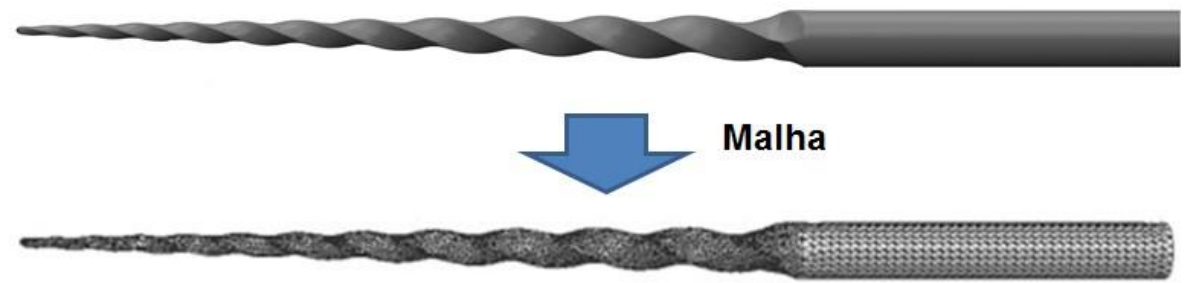

Figura 3. Esquema da elaboração do modelo de elementos finitos para a lima ProTaper Universal F1.

O número total de elementos foi ecolhido com base em pré-testes de flexão até $45^{\circ}$ para verificar a convergência de malha. A Figura 4 exibe o resultado destes testes, onde foram utilizadas quatro malhas com diferentes números de elementos. Neste gráfico é mostrada a força necessária para fletir o instrumento até $45^{\circ}$ versus 0 número de elementos utilizados. A malha com 51.184 elementos apresentou a melhor combinação entre confiabilidade e tempo de processamento.

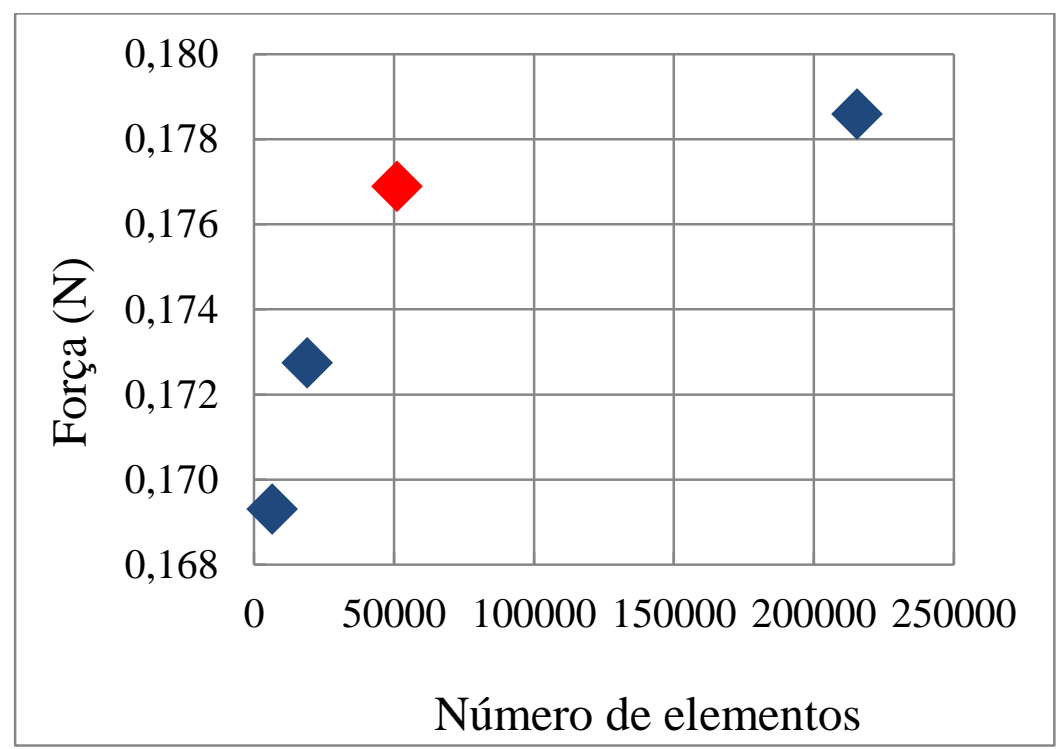

Figura 4. Resultados dos testes de convergência de malha.

As curvas tensão-deformação para os fios de $\mathrm{Ni}$-Ti convencional e $\mathrm{Ni}$-Ti M-Wire obtidos durante os ensaios de tração até a ruptura são exibidas na Figura 5, enquanto na Tabela 1 são mostrados os valores dos parâmetros necessários como dados de entrada para a elaboração do modelo constitutivo. A forma das curvas pode ser explicada da seguinte maneira: Inicialmente o material encontra-se no

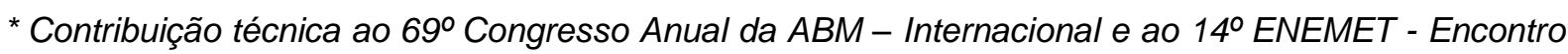
Nacional de Estudantes de Engenharia Metalúrgica, de Materiais e de Minas, 21 a 25 de julho de 2014, São Paulo, SP, Brasil. 


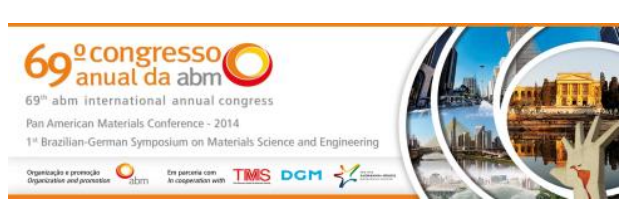

estado austenítico (fase mais estável nesta temperatura); uma vez submetido a tensão, o material experimenta a deformação elástica da austenita (regime linear inicial das curvas) até atingir o chamado plateau superelástico, onde observa-se grande deformação e quase nenhum aumento no valor na tensão; neste plateau ocorre a transformação martensítica induzida por tensão; a fase martensita originada deste processo é deformada elasticamente com o aumento da carga (segundo regime linear das curvas) até atingir o regime plástico e eventual ruptura. Antes de atingir o regime plástico, em qualquer ponto, a retirada da carga ocasionaria a transformação de fase reversa, e recuperação de toda a deformação. Neste trabalho não há interesse no retorno da curva de tração, uma vez que a análise numérica ocorre no material sob condições críticas de carregamento e sob tensão. Assim, curvas de tração até a ruptura do material são suficientes para esta análise numérica.

Nota-se que o plateau superelástico para ambos os fios encontra-se em níveis aproximados de tensão, porém o fio M-Wire apresenta maior elongação total e menores valores de módulo elástico para ambas as fases. Isso indica que de fato, o tratamento termomecânico utilizado na liga Ni-Ti M-Wire afeta suas propriedades mecânicas.

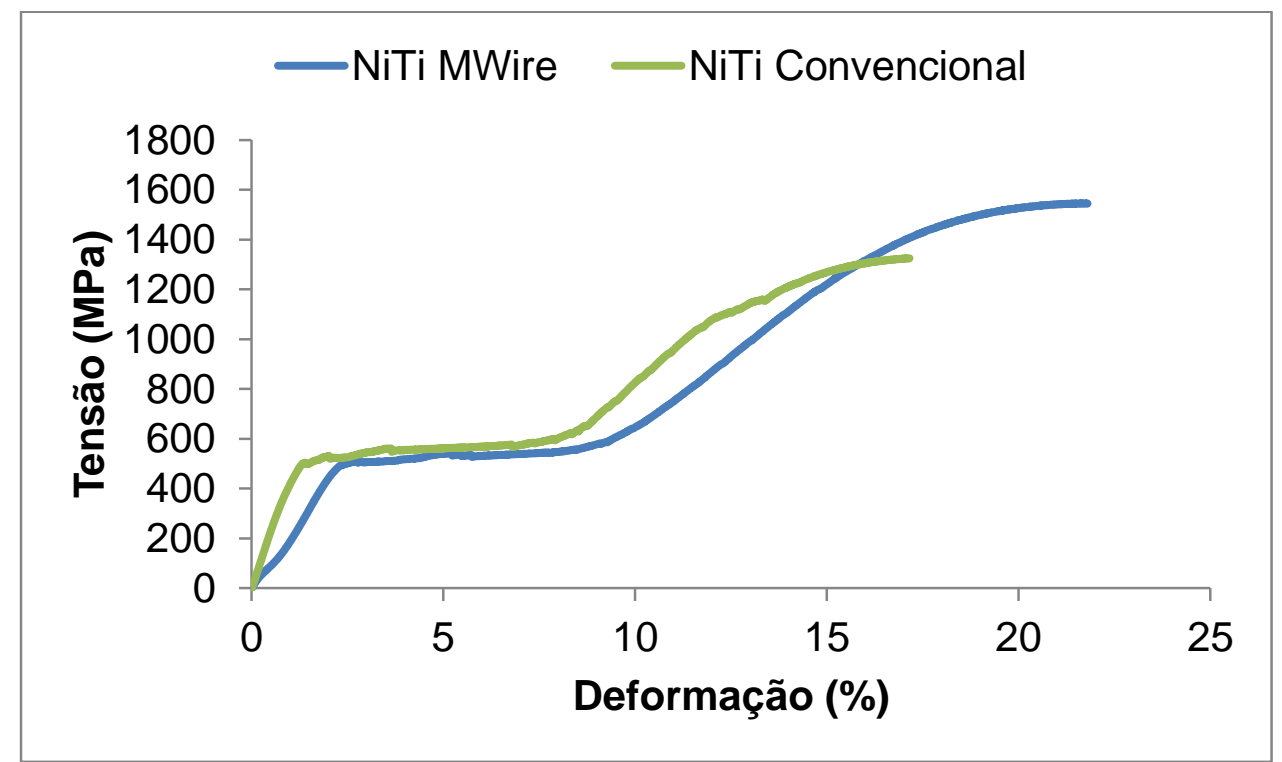

Figura 5. Curvas tensão-deformação para os fios Ni-Ti convencional e Ni-Ti M-Wire.

Tabela 1. Parâmetros utilizados para a elaboração do modelo constitutivo

\begin{tabular}{cccc}
\hline Parâmetros & Descrição & Convencional & M-Wire \\
\hline$E_{A}$ & Módulo de elasticidade da austenita & $43900 \mathrm{MPa}$ & $24600 \mathrm{MPa}$ \\
$v_{A}$ & Razão de Poisson da austenita & 0,33 & 0,33 \\
$E_{M}$ & Módulo de elasticidade da martensita & $12800 \mathrm{MPa}$ & $11000 \mathrm{MPa}$ \\
$v_{M}$ & Razão de Poisson da martensita & 0,33 & 0,33 \\
$\varepsilon^{L}$ & Patamar superelástico & $7 \%$ & $7 \%$ \\
$(\delta \sigma / \delta T)_{L}$ & $(\delta \sigma / \delta T)$ durante a carga & 6,7 & 6,7 \\
$\sigma_{L}^{S}$ & Tensão de início de transformação & $500 \mathrm{MPa}$ & $500 \mathrm{MPa}$ \\
$\sigma_{L}^{E}$ & Tensão de fim de transformação & $580 \mathrm{MPa}$ & $570 \mathrm{MPa}$ \\
$T_{0}$ & Temperatura & $22^{\circ} \mathrm{C}$ & $22^{\circ} \mathrm{C}$ \\
\hline
\end{tabular}

* Contribuição técnica ao $69^{\circ}$ Congresso Anual da ABM - Internacional e ao 14ํㅡㄹ ENEMET - Encontro Nacional de Estudantes de Engenharia Metalúrgica, de Materiais e de Minas, 21 a 25 de julho de 2014, São Paulo, SP, Brasil. 


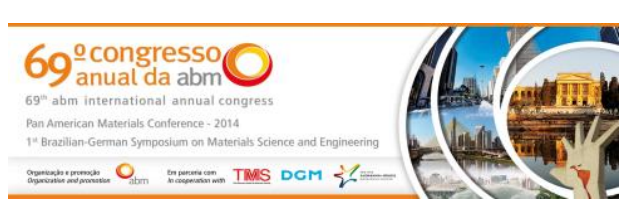

As curvas de momento vs. deslocamento durante a flexão até $45^{\circ}$, calculadas pela simulação numérica, são apresentadas na Figura 6. Para todos os valores de inclinação, o instrumento de Ni-Ti M-Wire apresentou menores valores de momento, ou seja, esta liga exige menores forças para que o instrumento seja fletido em comparação com um instrumento feito de $\mathrm{Ni}$-Ti convencional. Essas diferenças são observadas principalmente em valores de flexão mais baixos $\left(<30^{\circ}\right)$. Porém, para valores acima de $30^{\circ}$ de flexão, as diferenças se estreitam e os valores de momento tendem a se aproximar para ambas as limas. Esses resultados estão de acordo com o trabalho publicado por Pereira et al. [10], que realizaram ensaios de flexão em 3 pontos em fios convencionais e M-Wire. Os resultados apontam o fato de que em pequenas deflexões, os fios M-Wire apresentam valores de momento bem abaixo quando comparados com os valores para os fios de Ni-Ti convencional. Porém, com o aumento da deformação, as diferenças se estreitam e é observado até mesmo uma inversão em grandes deformações. De toda a forma, para valores intermediários de flexão, o instrumento confeccionado a partir da liga Ni-Ti M-Wire demanda menores esforços para deformação. Isso é um indicativo de que os danos estruturais ocasionados pela rotação sob flexão, e eventual fadiga, são menores nos instrumentos de Ni-Ti M-Wire. A não linearidade das curvas está relacionada com o elevado grau de deformação aplicada e principalmente com a complexidade geométrica do instrumento.

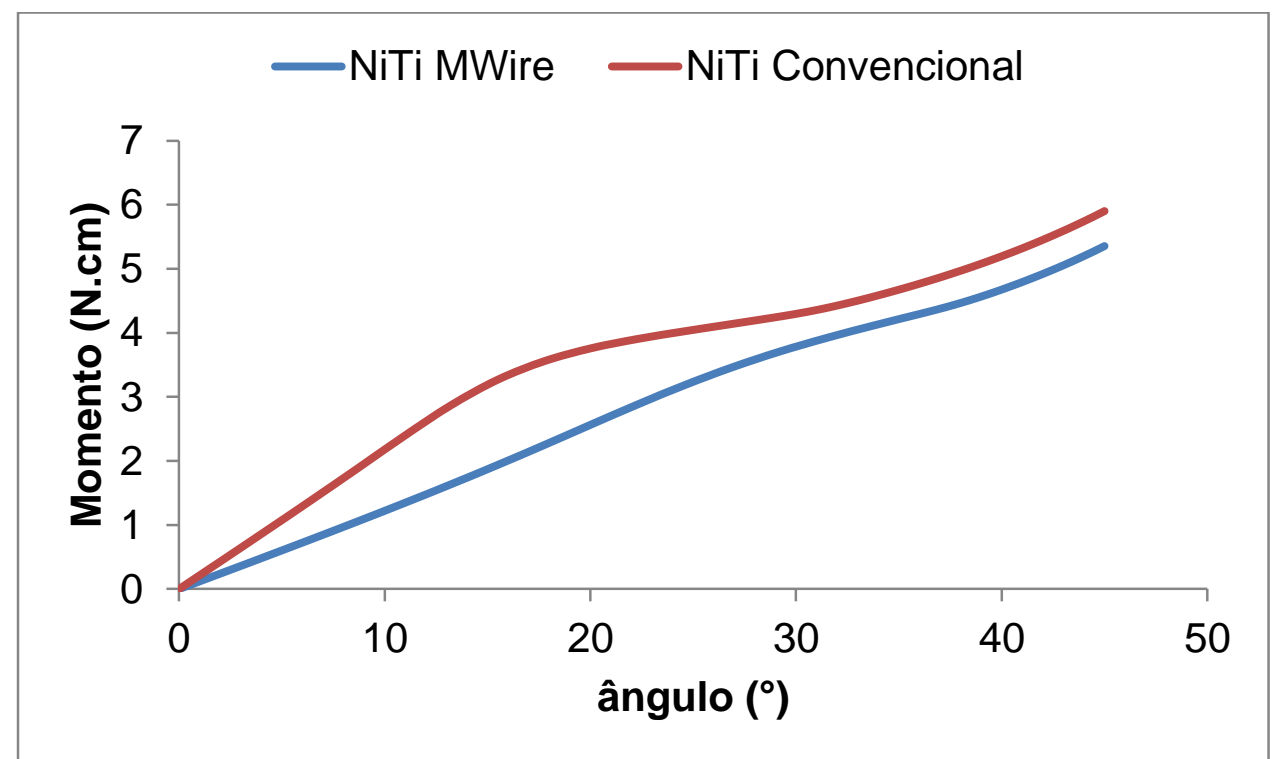

Figura 6. Curvas momento vs. deslocamento em flexão para as limas de Ni-Ti convencional e M-Wire obtidas a partir da simulação numérica.

É importante frisar-se que não seria possível realizar esta análise experimentalmente, uma vez que instrumentos feitos a partir de $\mathrm{Ni}-\mathrm{Ti} \mathrm{M}$-Wire disponíveis comercialmente apresentam outras geometrias, diferentes daquelas encontradas em instrumentos feitos a partir de $\mathrm{Ni}$-Ti convencional. Assim, eventuais diferenças nos resultados também seriam consequência da influência de diferentes geometrias. Este fato evidencia uma grande vantagem em trabalhar-se com simulação numérica.

A distribuição de tensões, segundo o critério de von Mises, nos instrumentos fletidos é exibida na Figura 7. Nota-se que há pouca diferença em termos de valores de

* Contribuição técnica ao 69 Congresso Anual da ABM - Internacional e ao 14ํㅡㄹ ENEMET - Encontro Nacional de Estudantes de Engenharia Metalúrgica, de Materiais e de Minas, 21 a 25 de julho de 2014, São Paulo, SP, Brasil. 


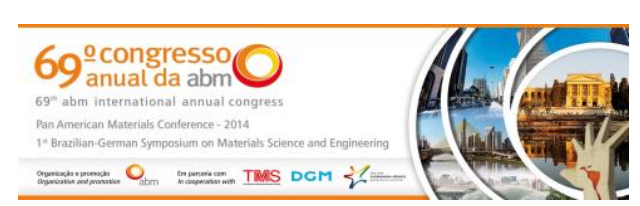

direta com os valores de tensão a que estes instrumento são sujeitos quando fletidos. Quanto maior a tensão aplicada, maior o risco de fadiga em serviço.

\section{Agradecimentos}

Os autores agradecem à Universidade de Zaragoza, Espanha, pelas medidas de micro-tomografia. Este trabalho foi parcialmente financiado pela Coordenação de Aperfeiçoamento de Pessoal de Nível Superior (CAPES/PROEX); Conselho Nacional de Desenvolvimento Científico e Tecnológico (CNPq) e Pró-reitora de Pesquisa da Universidade Federal de Minas Gerais.

\section{REFERÊNCIAS}

1 Petrini L, Necchi S, Taschieri S, Magliavacca, F. Numerical study on the influence of material characteristics on $\mathrm{Ni}-\mathrm{Ti}$ endodontic instrument performance. Journal of Materials Engineering and Performance. 2009; 18(5-6):631-637.

2 Walia H, Brantley WA, Gerstein HN. A initial investigation of the bending and torsional properties of nitinol root canal files. Journal of Endodontics. 1988; 14 (7):346-351.

3 Glosson CR, Haller RH, Dove SB, del Rio CA. Comparison of root canal preparations using NiTi hand, NiTi engine driven and K-Flex endodontic instruments. Journal of Endodontics. 1995; 21:146-151.

4 Otsuka K, Wayman CM. Shape Memory Materials. Cambridge University Press, Cambridge, UK, 1988.

5 Pruett J, Clement D, Carnes DJ. Cyclic fatigue testing of nickel-titanium endodontic instruments. Journal of Endodontics. 1997; 23(2)77-85.

6 Melo MCC, Bahia MGA, Buono VTL. Fatigue resistance of engine-driven rotary nickeltitanium endodontic instruments. Journal of Endodontics. 2002; 28(11):765-769.

7 Bahia MGA, Dias, RF, Buono, VTL. The influence of high amplitude cyclic straining on the behaviour of superelastic NiTi. International Journal of Fatigue. 2006; 28(9):10871091.

8 Figueiredo AMG, Modenesi P, Buono VTL. Low-cycle fatigue life of superelastic NiTi wires. International Journal of Fatigue. 2009; 31:751-758.

9 Bahia MGA, Gonzalez BM, Buono VTL. Fatigue behaviour of nickel-titanium superelastic wires and endodontic instruments. Fatigue \& Fracture of Engineering Materials \& Structures. 2006;29(7):518-523.

10 Pereira ESJ, Gomes RO, Leroy, AMF, Singh R, Peters AO, Bahia MGA, Buono VTL. Mechanical behavior of M-Wire and conventional NiTi wire used to manufacture rotary endodontic instruments. Dental Materials. 2013; 29:318-324.

11 Auricchio F, Petrini L. A three-dimensional model describing stress-temperature induced solid phase transformations: solution algorithm and boundary value problems. International Journal of Numerical Methods in Engineering. 2004; 61:716-737.

12 International Organization for Standardization ISO 3630-1. Dental root-canal instruments- Part 1: files, reamers, barbed broaches, rasps, paste carriers, explorers and cotton broaches. Switzerland: International Organization for Standardization, 1992.

13 Santos LA, López JB, Las Casas EB, Bahia MGA, Buono VTL. Mechanical behavior of three nickel-titanium rotary files: a comparison of numerical simulation with bending and torsion tests. Materials Science and Engineering C. 2014; 37:258-263.

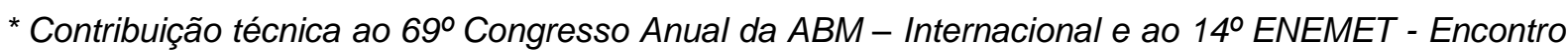
Nacional de Estudantes de Engenharia Metalúrgica, de Materiais e de Minas, 21 a 25 de julho de 2014, São Paulo, SP, Brasil.
} 